\title{
Philosophiques
}

\section{La métaphysique en dialogue}

Après six méditations métaphysiques quelques responsiones

\section{Jean Grondin}

Volume 41, numéro 2, automne 2014

URI : https://id.erudit.org/iderudit/1027230ar

DOI : https://doi.org/10.7202/1027230ar

Aller au sommaire du numéro

Éditeur(s)

Société de philosophie du Québec

ISSN

0316-2923 (imprimé)

1492-1391 (numérique)

Découvrir la revue

Citer ce document

Grondin, J. (2014). La métaphysique en dialogue : après six méditations métaphysiques quelques responsiones. Philosophiques, 41(2), 403-412. https://doi.org/10.7202/1027230ar d'utilisation que vous pouvez consulter en ligne.

https://apropos.erudit.org/fr/usagers/politique-dutilisation/ 


\title{
La métaphysique en dialogue
}

\section{Après six méditations métaphysiques quelques responsiones}

\author{
JEAN GRONDIN
}

\author{
À la mémoire d'Yvon Lafrance (I930-2014), Ami, \\ grand lecteur critique, bâtisseur et fondateur de la \\ revue Philosophiques.
}

C'est un bonheur et un honneur inespérés que de répondre ici à des lectures aussi pénétrantes de mon ouvrage sur "L'idée de la métaphysique ». À vrai dire, et sans fausse modestie, je ne m'attendais pas à ce que cet ouvrage trouve des lecteurs. C'est qu'il s'efforce de penser ensemble la métaphysique, son principal sujet, et l'herméneutique. Ces deux disciplines me passionnent depuis toujours, mais je sais que les métaphysiciens ne s'intéressent pas beaucoup à l'herméneutique, et que les herméneutes entretiennent souvent une sainte horreur de la métaphysique. Voir le terme de métaphysique dans un titre, surtout s'il n'est pas précédé d'un "post-» ou d'un «non- », suffira à les détourner. Mais je n'y peux rien, la métaphysique incarne pour moi un effort de compréhension. Elle l'a toujours été et le restera toujours. Aristote l'exprime clairement quand il introduit à son idée de la métaphysique en disant que «tous les hommes aspirent par nature à comprendre» (si l'on peut modifier la traduction d'un texte archiconnu, sans faire violence à son sens). Quant à l'herméneutique, elle aime insister sur les thèmes de la compréhension et de l'interprétation, au point d'y voir des propriétés essentielles de l'animal autointerprétant (Taylor) que nous sommes. La métaphysique n'affirme-t-elle pas la même chose quand elle souligne que l'homme se distingue par sa raison, celle-ci n'étant rien d'autre que l'effort de comprendre les raisons des choses? L'herméneutique repose ainsi sur un sol métaphysique. Cela est non seulement vrai de sa méthode ou façon de faire, l'activité de l'interprétation, mais aussi de son objet. Que s'agit-il d'interpréter en herméneutique? Des textes, bien sûr, mais d'abord et surtout des choses et des personnes. Je peux comprendre - ou ne pas comprendre, mais cela présuppose l'effort de compréhension - quelqu'un ou quelque phénomène. L'herméneutique, du moins telle que je la défends, s'élève ainsi contre l'idée selon laquelle le monde et autrui seraient foncièrement inintelligibles. J'y vois une idée fausse et pour tout dire déprimante, bien que répandue et inlassablement ressassée par les râleurs de la condition humaine que sont souvent devenus aujourd'hui les humanistes. Un humaniste, c'est au contraire pour moi quelqu'un qui croit en l'humain et qui affirme ses capacités infinies de comprendre le monde dont témoignent la science, les arts et les réalisations historiques de l'humanité qui la tirent de l'ignorance et de la barbarie. Cette compréhension est forcément herméneutique, et comme ce qui est compris, c'est l'être, l'être peut effectivement être compris (en langage, ajoute Gadamer). À mes yeux, il est moins 
essentiel, quoique important, de mettre en relief l'idée que cette compréhension s'effectue en langage que de dire que l'être peut bel et bien être compris et que nous pouvons en pénétrer les raisons. Une science ou une philosophie qui le nierait se tirerait une balle dans le pied, comme le dit la jolie formule anglaise. À mon sens, des conséquences plus métaphysiques au sujet de la rationalité du monde (entendons, de son intelligibilité de principe) et de celle de l'homme peuvent en être tirées. La métaphysique l'a en tout cas toujours fait. Je m'inscris humblement dans cette tradition. Humblement (et sans fausse humilité encore une fois) parce que je ne suis pas sûr d'avoir une théorie métaphysique absolument originale et renversante à proposer - il faut être prétentieux pour proposer quelque chose de tel -, et que ma tâche est avant tout de transmettre la pensée métaphysique et l'exigence incontournable qu'elle continue de représenter pour la philosophie et la science ellemême. Il est des savoirs qui méritent d'être transmis et qui pourraient se perdre s'ils ne l'étaient pas. Or ce qu'il y a lieu de transmettre, en cet âge herméneutique de la pensée, ce n'est pas une doctrine massive, ou pire, une idéologie, c'est un dialogue (idée herméneutique, si l'on veut), le dialogue de longue durée sur le sens des choses qu'a toujours été la métaphysique. C'est cette idée que je soumettais à la discussion et contre toute attente elle a trouvé des lecteurs attentifs dans les contributions ici réunies. J'aimerais d'abord et surtout les remercier de leur lecture, car sans lecture, et sans lecture personnelle où l'on engage sa propre pensée et son expérience de vie, il n'est pas de métaphysique. Et c'est tant mieux si la lecture est aussi une idée herméneutique. En métaphysique, ce qui compte, Socrate le disait, ce n'est pas la provenance des idées, mais qu'il y en ait, et des bonnes. Disputons donc, sans trop nous disputer.

Par qui ou quoi commencer? Vieux problème métaphysique, que je tranche ici en suivant la raison alphabétique. La contribution remarquable de Rémi Brague souligne avec justesse que, sur la question du génie du platonisme et tant d'autres sujets essentiels, nous sommes en sympathie fondamentale. Cela tient au fait que Rémi Brague fait partie des rares auteurs contemporains dont je pense bien avoir lu tous les livres. C'est qu'ils sont immensément instructifs, souvent drôles (la philosophie ne gagne rien à se prendre trop au sérieux), nagent hardiment à contre-courant des idées reçues et regorgent d'allusions à mes bandes dessinées préférées. Nos différences sont toujours infinitésimales. Toute sa pensée, qui aura été davantage marquée par Leo Strauss, cherche à "modérer l'enthousiasme de la Modernité pour elle-même ", voire à effectuer, avec un goût vorace pour la provocation, un «retour au Moyen Âge ${ }^{1}$. En Nord-Américain impénitent, j'adopte peut-être une attitude moins soupçonneuse que la sienne quand il s'agit de la science contemporaine et de la modernité, dont je ne regrette que 
le nominalisme débridé que je tiens, pour parler comme Hegel, pour une crispation de l'entendement.

Rémi Brague commence par se demander si la science nous permet vraiment de comprendre les phénomènes. C'est qu'à ses yeux la science moderne permettrait "plutôt de les décrire dans ce langage mathématique dans lequel Galilée nous a appris que la nature était rédigée, d'en formuler les lois, et partant, d'en prévoir la répétition à l'identique partout où les conditions de l'expérimentation sont les mêmes ". Il se réclame ici d'Auguste Comte qui a dit que la recherche des causes, dont il offre une version caricaturale (ce qui est aussi vrai de sa conception de la métaphysique), nous aurait été refusée, mais qu'elle aurait été remplacée et compensée par celle des lois qui nous permettent d'observer des relations constantes entre les phénomènes. Auguste Comte (mort en I 857) a-t-il vraiment dit le dernier mot à propos de l'essence de la science moderne? Je ne le crois pas. D'une part, il me semble évident que la connaissance des lois permet aussi d'expliquer la cause des phénomènes. Pour prendre un exemple trivial, la loi de l'offre et de la demande en économie permet généralement de comprendre pourquoi le prix d'un article baisse ou augmente. L'explication par des lois représente le plus souvent un adjuvant à la compréhension. C'est un grand herméneute, Paul Ricœur, qui l'a dit, assez simplement: expliquer plus, c'est comprendre mieux. D'autre part, je ne suis pas sûr que la science se contente toujours de décrire en langage mathématique les lois de la nature. Je trouve qu'il est très utile qu'elle le fasse (comme Natorp, j'y vois même une réaffirmation par d'autres moyens du primat métaphysique de l'eidos chez Platon). Il ne faut pas toujours croire ce que les épistémologues ou les philosophes comme Comte racontent de la science et de la pratique réelle des scientifiques (lesquels n'ont pas non plus perdu le sens des causes finales comme voudrait le faire croire une certaine historiographie, elle-même non dépourvue de visées téléologiques). Je me souviens d'avoir vu un jour mon père opérer un patient qui souffrait d'un anévrisme de l'aorte abdominale, qui est une dilatation subite de l'artère pouvant conduire à sa rupture et à une mort à peu près certaine. Avant d'opérer le patient, le médecin a d'abord dû comprendre et identifier le phénomène. Je ne sais pas si la mathématique a été ici d'un grand secours (elle l'est souvent et c'est tant mieux pour elle comme pour nous), la reconnaissance compréhensive du général l'a certainement été, et le patient fut sauvé. Si j’avais été à sa place, j'aurais été très reconnaissant envers la science moderne et ses capacités de compréhension. On aura remarqué (Peter Obadachian l'a justement fait) que les exemples médicaux abondent dans mon livre. C'est que l'art médical a toujours représenté pour moi, peut-être pour des raisons biographiques, le modèle par excellence de la science. On déplore souvent, sans doute pour de bonnes raisons par ailleurs, la place qu'occupent la froide mathématique et les données abstraites en médecine, mais, d'après mon expérience, la pratique de la médecine révèle qu'il s'agit d'une science franchement herméneutique, compréhensive et 
humaine: elle est confrontée à des phénomènes, ou des symptômes, qu'elle s'efforce de comprendre et de guérir pour notre plus grand bien (qu'il me soit permis d'y voir sa cause finale). Pour y parvenir, toutes les explications sont bonnes.

$\mathrm{R}$. Brague en vient à me poser la question précise: comment articuler l'une sur l'autre la science moderne et la technologie qu'elle rend possible, d'une part, et d'autre part l'herméneutique? Ma réponse se ferait en deux temps, que j'énonce en style télégraphique (ou twitteresque parce qu'il n'y a plus beaucoup de télégraphes). I) Ce travail a déjà été accompli, en partie, par Gadamer, quand il s'est efforcé de justifier philosophiquement des expériences de vérité que ne permettrait pas d'embrasser le modèle de la science méthodique moderne. L'apport de Gadamer est ici admirable, et je lui reprocherais seulement de ne peut-être pas avoir assez défini ce qu'il entendait par méthode. C'est qu'il est aussi parti d'une idée philosophique assez générale et non de la pratique effective (donc de la méthode au sens plein) des sciences exactes. 2) La deuxième partie de ma réponse consisterait justement à mettre l'accent sur la dimension herméneutique de la science elle-même, évidente dans ses tâtonnements incessants, ses remises en question, parfois radicales, son caractère dialogique et coopératif, son imagination quand vient le temps d'élaborer des hypothèses, son expérience du conflit des interprétations, pour ne rien dire de ses paradigmes, qui l'inscrivent dans un travail de l'histoire, ou de son élément rhétorique parce que Kuhn et d'autres en ont traité. Je ne parlerais donc pas d'une schizophrénie entre «la manière dont nous pouvons vivre notre humanité, individuellement et collectivement» et "ce que nous savons du monde matériel». Je comprends ce que Rémi Brague veut dire: comme le dernier Husserl il estime que les explications scientifiques semblent s'être éloignées du monde de la vie. Mais cet écart tient à un manque d'éducation de la part des béotiens scientifiques que nous sommes si souvent. À mon sens, une découverte scientifique peut toujours être comprise et traduite dans un langage que nous pouvons comprendre et appliquer au monde de notre vie. C'est l'un des principes directeurs de l'herméneutique.

Sur la question du nominalisme, ma réponse courte consisterait à dire qu'il me faudrait faire une petite histoire du nominalisme. J'ai indiqué mes sources ailleurs ${ }^{2}$, mais ne voudrais pas ici que m'auto-citer. Lorsque je parle du nominalisme, je vise toujours le constructivisme contemporain, l'idée que tout sens relève d'une construction culturelle qui ne dépend que du constructeur et de ses orientations idéologiques. Elle remonte aux origines de la science moderne et à la conception du réel construite par les nominalistes médiévaux, que je ne vise pas directement et dont on sait que le motif était théologique: un royaume d'essences prédéterminées étant pour eux

2. Heidegger et le défi du nominalisme, dans Les Temps modernes 63 (2008), n 650, $235-247$. 
incompatible avec la toute-puissance divine, il n'y a que des êtres individuels dont nous observons les régularités.

Pour ce qui est de Kant, le grand mérite que je lui reconnais est d'avoir vu et été le dernier à voir dans la doctrine du souverain Bien, d'origine platonicienne et aristotélicienne, le principe qui détermine la fin ultime de notre raison (suivant le titre de la section de la CRP où il répond aux trois grandes questions de la raison, et surtout à la question: que m'est-il permis d'espérer ?). Le souverain Bien dérivé qui correspond à l'accord entre la moralité de notre agir et le bonheur qu'elle pourrait se mériter dépend du souverain Bien originaire, l'intelligence supérieure seule capable d'évaluer la teneur morale de nos actions. C'est un souverain Bien selon la raison pratique, dont la formulation m'apparaît plus claire, elle est aussi beaucoup plus leibnizienne, dans la première que dans la deuxième Critique, dont Rémi Brague a raison de dire qu'elle glorifie exagérément la Loi morale au détriment du Bien. La première Critique respecte davantage le sens des choses.

Envers Théodore Geraets j'ai une dette incommensurable dont il n'a pas conscience. C'est qu'il fut l'organisateur, dans les années 70 , de colloques tenus à Ottawa où il invitait les plus grandes sommités philosophiques du temps, dont Gadamer, Ricœur, Habermas et Apel. La première fois que j'ai parlé à Gadamer et à Habermas, ce fut à Ottawa à l'occasion d'un de ces colloques sur la rationalité, donc grâce à Théo Geraets. Sans ces rencontres, je ne sais trop ce que je serais devenu. Sa contribution a le bonheur de nous plonger dans une lecture herméneutique de Hegel où je reconnais l'apport précieux de Gadamer et de Merleau-Ponty. Je ne débattrai pas ici de la question de savoir si Hegel était ou non un métaphysicien. Pour moi c'est la chose la plus évidente du monde, et la réticence d'hégéliens comme Jaeschke tient plus à leurs affects anti-métaphysiques (c'est un effet de la métaphysique nominaliste) qu'à l'œuvre de Hegel lui-même dont le «système" (toujours en mouvement, Théo Geraets l'a mis en évidence avec grande finesse) se divise en logique, philosophie de la nature et philosophie de l'esprit. Y a-t-il des logiques et des philosophies de la nature plus métaphysiques que celle de Hegel? Quant à sa philosophie de l'esprit, elle se termine sur le texte, cité en grec, de la Métaphysique d'Aristote qui présente la pensée divine comme pensée du souverain Bien (tou aristou, tou malista, I072 b I9) et dès lors comme pensée de la pensée. Si cette pensée n'est pas métaphysique, il n'y a jamais eu de métaphysique.

Théo Geraets, comme Guillaume St-Laurent, a raison de dire que ma lecture un peu métaphysique de l'herméneutique va sans doute au-delà des intentions de Gadamer. Je plaide coupable s'il s'agit d'un crime. Mon propos n'est pas de rompre avec la phénoménologie (G. St-Laurent), mais de tirer les conséquences de la critique du nominalisme que l'on trouve dans la dernière partie de Vérité et méthode, et de l'idée selon laquelle il y a un langage des choses, une logique de l'être si l'on préfère, que nous pouvons tenter de comprendre. "L'être qui peut être compris est langage », dit Gadamer. 
Dans cette déclaration, Gadamer préfère insister sur l'idée que c'est dans le langage que se déploient notre compréhension et cette intelligibilité des choses. Je le reconnais sans peine, mais j'insisterais pour ma part sur l'intelligibilité des choses qui est signifiée par là: l'être - surprise! - peut être compris! Idée einsteinienne que j'oppose volontiers aux prophètes de l'incompréhensibilité foncière du monde. Théo Gearets l'a bien senti. Il n'a pas tort de dire que les conséquences théologiques que l'on peut en tirer ne sont probablement pas celles de Gadamer. Mais Gadamer peut-il défendre son idée sans avoir recours à la métaphysique? Heidegger était de ceux qui pensaient que non. Il le disait bien entendu pour critiquer Gadamer. Je le dis pour ma part afin que l'herméneutique assume sa cohérence.

Ce problème resurgit dans la lecture attentive de la métaphysique que propose le fin spécialiste de la métaphysique du Dasein qu'est François Jaran. Il y distingue à bon droit deux concepts, que j'appellerais superposés et qui sont sans doute détachables, de la métaphysique et qui correspondent au doublet de la metaphysica generalis, strictement ontologique, et de la metaphysica specialis, qui s'avance sur le terrain du théologique. Je pense qu'il est d'accord avec moi pour dire qu'il y a en toute philosophie digne de ce nom une métaphysique au sens où on y trouve une théorie générale sur l'être, et ses raisons, et pour dire qu'on ne peut réfuter ce projet métaphysique que par une autre métaphysique. C'est l'une des thèses du livre qui cherche à défendre le caractère fondamental et incontournable de la réflexion métaphysique. Vue sous cet angle, une ontologie purement matérialiste est aussi une métaphysique, et elle fait partie de ce que j'appelle le canon de la métaphysique, le long dialogue de l'humanité sur le sens des choses (qui repose aussi sur la materia des choses - Aristote y a toujours aperçu une cause fondamentale —, materia qu'il importe cependant de comprendre à partir de la «maternité» platonicienne de la chôra, présupposant qu'il y a une causalité de l'eidos ${ }^{3}$ ). M. Jaran a bien vu que je donnais, dans la dernière leçon, un tour plus métaphysique à cette quête philosophique des fondements quand j'abordais et réaffirmais l'horizon théologique de la métaphysique. François Jaran me demande si cela implique que toute philosophie doive admettre une transcendance. Bien sûr que non, car on se priverait alors des lumières de plusieurs philosophies. Mon idée est plutôt: I) qu'il y a des raisons (la métaphysique est un discours rationnel) qui permettent d'affirmer la transcendance (les miennes se situent dans la continuité de la tradition métaphysique), et 2) que toute philosophie doit comporter un horizon de transcendance, qui n'a pas à être théologique, mais qui nous explique pour quoi la vie mérite d'être vécue et réponde à la question: que m'est-il permis d'espérer? Nous vivons à une époque de métaphysique nominaliste, où la transcendance est devenue pour plusieurs infréquentable. C'est une situation que je comprends et que je m'efforce de décrire, non sans

3. Du sens des choses, p. I30. 
souligner que la foi dans le nominalisme nous coupe alors du plus clair de notre tradition philosophique, devenue désuète.

François Jaran fait aussi valoir que des philosophies a-métaphysiques sont possibles. Il est vrai que tout en philosophie n'est pas métaphysique: on peut s'intéresser à des questions logiques, épistémologiques, esthétiques, etc. qui n'ont pas à être métaphysiques (même si leurs fondements le sont souvent). Husserl en est-il un bon exemple? Il pensait que oui. J'évoque son exemple avec d'autant plus d'empathie que j'ai pensé à son titre «L'idée de la phénoménologie» en intitulant ce livre «L'idée de la métaphysique» (l'autre sens du titre était l'allusion à l'eidos tel que l'entend la métaphysique). Ne pas faire de la métaphysique veut dire pour lui qu'on ne se prononce pas sur l'être des choses et que l'on se contente de décrire les phénomènes tels qu'ils apparaissent à la conscience. Cette description estelle toujours aussi a-métaphysique qu'il le croit? Ma vision a été imprégnée par Heidegger qui a vu dans cette notion de conscience une décision métaphysique lourde de conséquences. Il est certainement possible et sans doute plus fécond de lire Husserl autrement. Mais qu'est-ce qui fait qu'une description des phénomènes mérite d'être dite philosophique? N'est-ce pas le fait qu'elle s'interroge sur les raisons des phénomènes? Si on veut se contenter d'une description immanente, on pourrait lui préférer celles de la littérature, de la poésie, voire des sciences cognitives. Une description philosophique doit nous aider à comprendre, et elle ne le peut sans quelque métaphysique.

Peter Odabachian a la généreuse perspicacité de situer ma contribution dans la continuité de l'Introduction à la métaphysique, publiée en 2004, et de souligner que ce titre se comprenait comme une réponse au livre du même nom de Heidegger, auquel je dois beaucoup, mais dont je ne partage pas l'aversion antimétaphysique que j'estime caricaturale (et auto-contradictoire pour un auteur qui veut réveiller la question de l'être). M. Odabachian me reproche discrètement de faire trop peu de cas de la mortalité. Je suis d'accord avec lui pour dire qu'elle est l'énigme fondatrice de notre humanité et qu'elle ne peut pas ne pas être présupposée quand on cherche à répondre à la question "quel sens m'est-il permis d'espérer?». J'en avais parlé davantage dans un essai antérieur, $D u$ sens de la vie (2003), et mon grand ami Yvon Lafrance m'avait alors reproché de trop en parler. C'est que - c'est une autre raison qui explique peut-être le silence relatif de l'essai plus récent sur la question - notre propre mort ne fait pas partie de notre expérience personnelle. Ricœur y a beaucoup insisté dans ses écrits et préférait suivre Spinoza quand il disait que l'homme libre était celui qui ne pensait jamais à la mort. J'en tire en tout cas la conclusion que je ne suis pas un homme libre...

Peter Obadachian me pose l'excellente question de savoir pourquoi notre époque a perdu le sens des choses et succombe si allègrement au constructivisme. Il faudrait que j'y réfléchisse davantage. Chose certaine, l'homme est volontiers rassuré par ce qu'il fabrique lui-même et ce qui vient confirmer ses opinions préconçues (cela se vérifie dans la tendance, sur les réseaux sociaux 
par exemple, à ne tenir compte que des avis de ceux qui pensent comme nous). La question du sens des choses l'invite à sortir de sa coquille et à s'ouvrir à ce qui ne se conforme pas à ses schémas préconstruits, idéologiques. Le constructivisme a aussi quelque chose d'enivrant: le réel est celui que je "construis». Vraiment? Je n'ai jamais eu le sentiment que c'était le cas.

Guilllaume St-Laurent a le mérite de soulever le problème épistémologique lancé, mais non résolu par Kant, du type de connaissance qu'est le savoir métaphysique. Mon ouvrage en étant un de métaphysique et non d'épistémologie, c'est une question dont j'ai peu traité. Mais puisque la métaphysique a affaire à la question de la vérité, c'est un thème qu'elle ne peut ignorer, même si pour elle la vérité est d'abord fondée dans la veritas rerum dont cherche à s'approcher notre connaissance. Le paradigme scientifique dominant voudrait nous faire croire qu'il n'y a que deux types de connaissance: le savoir d'opinion, purement subjectif, et le savoir scientifique, qui est le seul à pouvoir être dit objectif (Gadamer a bien montré ce que cela avait de fatal pour les sciences de l'esprit). La distinction entre la doxa et l'epistèmè remonte naturellement à Platon, mais il faut croire qu'elle n'avait pas tout à fait pour lui le sens qu'elle a pour nous: son modèle de la science, il le trouvait dans les mathématiques, et au-delà d'elles, dans la connaissance "métaphysique» des idées, plus rigoureuse parce qu'elle remontait à des principes derniers. Quant à l'opinion, il reconnaissait parfaitement qu'elle pouvait être dans le vrai. Notre distinction actuelle entre l'opinion et la science gagnerait donc à être nuancée. Il faudrait commencer par rappeler qu'en science tout ne relève pas de la seule certitude apodictique (pensons encore à Kuhn). Ce serait une tâche épistémologique intéressante que de distinguer les différents types de savoir en les disposant sur une échelle: à un extrême on pourrait situer les opinions les plus subjectives que l'on peut entretenir sur des questions de goût, dont on peut dire qu'elles sont purement "subjectives ", à l'autre le savoir mathématique, dont le prestige est inentamé depuis Platon puisqu'il permet une certitude apodictique, mais c'est vraisemblablement parce qu'il est analytique (pace Kant). Certains des vulgarisateurs de la «science», dont je redis qu'elle présente des visages et des types très divers, voudraient nous faire croire que son savoir est aussi certain que celui de la mathématique. Ce n'est pas le cas, même si elle peut s'en approcher. C'est que l'ensemble de notre savoir qui n'est pas mathématique, ou analytique, se fonde sur le langage et notre capacité discursive de comprendre les phénomènes. Ce savoir a toujours part à ce que l'herméneutique appelle la métaphoricité essentielle ou la nature rhétorique du langage. Ce savoir discursif peut être fondé et l'est de diverses manières selon les différents modes de savoir. La métaphysique fait partie de l'ordre du savoir. Je ne parlerais pas de "conviction» dans son cas, car cela la rapprocherait trop de la foi et de la religion (avec laquelle on la confond souvent), mais d'un savoir qui énonce ses raisons. L'argument e sensu rerum (qui n'est pas nouveau) dit que le sens ou la connaissance que nous avons du sens intelligent 
des choses autorise l'admission d'une intelligence supérieure. C'est pour moi et pour toute la tradition philosophique un argument très fort et dont on peut débattre, car on peut débattre de toute connaissance discursive.

Est-ce que cela revient à expliquer un mystère par une donnée plus mystérieuse encore, comme le croit Comte-Sponville dont s'inspire M. SaintLaurent? J'avoue que c'est l'argument de Comte-Sponville qui m'apparaît ici singulièrement faible. C'est que, en science, il n'y a rien d'exceptionnel à expliquer un phénomène par une hypothèse dont on ne sait rien ou peu de choses par ailleurs. C'est même la norme, une hypothèse étant toujours une "aventure hasardeuse ${ }^{4}$ ». Pensons à l'hypothèse courante du Big Bang: un physicien nous dira que c'est la meilleure hypothèse permettant d'expliquer les simulations mathématiques que l'on peut faire à propos de l'origine du monde, mais cela reste une affaire mystérieuse. De Dieu, nous savons peu de choses, mais je ne dirais pas que c'est une idée «mystérieuse ». En quoi l'estelle? C'est une idée que roo p. cent des mortels (ou 99.99 p. cent) comprennent et que l'on peut expliquer (par comparaison, l'homme de la rue ne sait pas trop ce qu'est la physique quantique ou la déconstruction, qui sont aussi des choses un peu mystérieuses). Les meilleurs philosophes l'ont toujours fait : Kant décrit cette idée comme un idéal nécessaire de la raison, Aristote en expose l'activité au livre XII de sa Métaphysique, Leibniz s'efforce même de justifier son action, et les premières parties des Sommes de Thomas y sont consacrées. Certes, c'est là le Dieu des philosophes (ou de la métaphysique). Je fais partie de ceux qui ne le dédaignent pas et estiment, avec la tradition métaphysique, qu'on peut y voir un sommet du savoir ou de la science humaine. D'aucuns peuvent lui préférer le Dieu des religions (ou des spiritualités parce que le terme de religion n'est plus très prisé). Il est très éloquent et se donne à connaître dans les textes sacrés dont je ne dirais pas non plus qu'ils sont mystérieux (Dieu est Amour, Dieu est le Logos, le créateur de toutes choses, etc.). Il y a aussi le Dieu des chefs-d'œuvre de l'art, le Messie de Händel, la Cène de Leonardo, les Confessions d'Augustin, voire le God de John Lennon ou l'Halleluja de Leonard Cohen, etc. L'idée de Dieu n'est pas mystérieuse (elle l'est en tout cas beaucoup moins que la bêtise humaine). L'argument e sensu rerum ne l'est pas non plus et a même le bonheur d'être accessible au commun des mortels: il est intelligent d'admettre une intelligence pour rendre compte de l'intelligence des choses. Ce qui est mystérieux, n'est-ce pas plutôt la répulsion que semble inspirer à certains toute pensée de la transcendance, pourtant essentielle à la nature humaine et à la philosophie? L'argument téléologique est parmi nous depuis plus de deux mille ans, et il le sera encore dans deux mille ans si l'humanité fait l'effort d'exister encore. Comme ce fut toujours le cas, il s'accompagnera immanquablement de contestations très honorables et qui font pour cette raison partie du fonds de la métaphysique. On me pardonnera de participer à ce débat, de penser

4. Modérément moderne, p. I76. 
qu'il mérite d'être poursuivi et de mettre en garde ceux qui voudraient le trancher de manière péremptoire. Ce n'est pas parce qu'une question aussi vitale ne peut être décidée comme une question d'arithmétique qu'elle ne nous procure pas un savoir, une connaissance et une sagesse.

Marc-Antoine Vallée a le flair herméneutique de se demander si l'herméneutique métaphysique que je cherche à rendre pensable n'obéit pas, sans s'en rendre tout à fait compte, au cercle herméneutique (lequel peut être vicieux): peut-on présenter la métaphysique comme un dialogue de longue durée sur le sens des choses, dialogue au sein duquel peuvent s'affronter des pensées opposées, et promouvoir en même temps une conception particulière de la métaphysique, inspirée du génie du platonisme? La question est très bonne. Je répondrais que c'est justement Platon qui permet cela: il ne fait guère de doute qu'il défend lui-même une métaphysique particulière, dont le principe des principes est l'idée du Bien qui irradie dans le sensible, mais cette métaphysique il la déploie toujours sous une forme dialogique et allusive. On trouve donc aussi chez lui une conception précise de la métaphysique et une mise en scène de la philosophie, dont Platon crée l'idée et l'élément, comme d'un dialogue entre interlocuteurs provenant de traditions diverses. Le cercle herméneutique opère toujours: on ne peut présenter ce long dialogue qu'est l'aventure métaphysique de l'humanité sans prendre part soi-même à cette conversation. La philosophie ne se pratique pas autrement et c'est en cela qu'elle est herméneutique.

Celui qui participe à cet entretien doit aussi s'efforcer de justifier ses positions ou sa situation herméneutique. C'est aussi une exigence platonicienne. M. Vallée estime que je justifie peut-être trop peu le statut des "essences » ou ce que j'appelle l'ordre d'intelligibilité au fondement de notre monde (le rappel de l'étymologie de materia a son importance dans ce contexte parce que la vulgate nominaliste contemporaine tient souvent la "matière» pour une réalité dernière et positive, oubliant qu'il s'agit d'une métaphore). Je pensais l'avoir au moins fortement suggéré en faisant souvent allusion à l'orientation nécessairement eidétique de la science, des arts et de la philosophie elle-même. Ces disciplines sont à même de penser le particulier, mais elles l'éclairent le plus souvent à partir de la supposition - métaphysique - de l'idée, qui laisse pressentir que l'ordre du monde a quelque chose d'intelligent et d'intelligible. Platon lui-même en a-t-il fourni des preuves définitives? Chose certaine, celui qui voudrait savoir comment s'effectue au juste la participation du sensible à l'intelligible, ou de quelle manière les idées "communiquent » à l'idée du Bien, ne trouvera pas d'explications très détaillées dans les textes de Platon. Le Phédon ( Ioo d) dit même que c'est là une question sur laquelle il ne veut rien décider, car l'essentiel est plutôt le coup d'œil sur le Beau lui-même qui resplendit assez dans le sensible pour se rappeler à notre admiration. Si quelqu'un a de meilleures raisons, je les adopterai volontiers. En métaphysique, comme en herméneutique, il n’y a pas de dernier mot. Le dialogue herméneutique se poursuit. 